\title{
Diagnostic anténatal : comportement médical face aux incertitudes diagnostiques et pronostiques
}

\author{
Antenatal diagnosis: medical behaviour faced to diagnostic and prognostic uncertainties
}

\author{
B. Langer $\cdot$ D. Mitanchez \\ (C) Springer-Verlag France 2013
}

Ce nouveau dossier thématique sur le diagnostic anténatal un peu plus d'un an après celui de juin 2012 nous a paru souhaitable pour d'une part aborder différents aspects de la prise en charge des couples dont le foetus est suspecté porteur d'une anomalie et d'autre part revenir sur le diagnostic prénatal non invasif (DPNI) sur sang maternel, dont le développement extrêmement rapide va conduire à un changement de paradigme en matière de dépistage et de diagnostic prénatal.

L'annonce d'une suspicion d'anomalie fœtale est toujours difficile comme nous le montre Luc Gourand. Par ailleurs, le diagnostic anténatal est aussi source d'incertitude (Marc Grassin). Incertitude pour le professionnel qui, dans certains dossiers, ne peut au mieux qu'annoncer un pourcentage de risque d'handicap, alors que les couples voudraient entendre des certitudes pour prendre une décision. Incertitude quant à la meilleure manière de délivrer des informations. Incertitude quant au degré de compréhension par les couples des informations données.

Aujourd'hui, en France, $96 \%$ des diagnostics de trisomie aboutissent à une interruption médicale de grossesse. AnneSophie Weingertner s'interroge sur les raisons de cette orientation. Jérome Gofette décrit les caractéristiques qui soustendent le concept d'eugénisme. Selon son analyse, il n'y a pas d'eugénisme dans le dépistage et le diagnostic anténatal développés en France.

La recherche de techniques moins invasives en diagnostic prénatal a fait l'objet de la plupart des recherches de ces dernières décades. Elle a abouti à la mise au point du dépistage combiné de la trisomie 21 fondé sur la mesure de la clarté nucale et le dosage des marqueurs sériques du $1^{\text {er }}$ trimestre. Ce dépistage, recommandé par l'HAS en 2007, est à

\footnotetext{
B. Langer $(\square)$

Département de gynécologie-obstétrique, hôpital de Hautepierre, F-67098 Strasbourg cedex, France

e-mail : Bruno.Langer@chru-strasbourg.fr

D. Mitanchez

Service de néonatologie, AP-HP, hôpital Armand-Trousseau, 26, avenue du Dr-Arnold-Netter, F-75571 Paris cedex 12, France
}

peine entré dans les habitudes en France qu'une révolution s'annonce, celle du dépistage faisant appel à l'ADN fotal circulant dans le sang maternel (Alexandra Benachi).

Le nombre impressionnant des travaux de recherche sur le DPNI, boostés par le fait qu'ils ont été pris en charge par le secteur commercial qui y voit d'importants bénéfices potentiels, fait que la revue Prenatal Diagnosis vient de leur consacrer deux tomes entiers (juin et juillet 2013). Aujourd'hui, de nombreuses questions se posent. Proposera-t-on le DPNI aux seules patientes classées à risque par le dépistage combiné ? Quand et comment la France se positionnera en matière de remboursement, alors que certains couples se sont déjà tournés aujourd'hui vers les propositions faites à l'étranger ou sur internet ? Le DPNI se limitera-t-il au dépistage des trois trisomies les plus fréquentes $(21,13$ et 18$)$ ou concernera-t-il tout le génome comme le proposent certaines compagnies américaines ? Bien sûr, il va générer de nombreuses questions d'ordre éthique ou sociétal. Ne va-t-il pas s'orienter vers un choix entre la poursuite ou l'interruption de la grossesse au lieu de développer la thérapie génique et les traitements in utero ? Il paraît essentiel d'insister d'emblée sur la nécessité de prévoir avec sa mise en place du temps de consultation et une éducation préalable des professionnels de santé si l'on veut maintenir le principe d'un consentement éclairé du couple.

En cas d'affection incurable et d'une particulière gravité, on accède à une demande des parents d'interrompre la grossesse. Pierre Betremieux présente la possibilité de réaliser des soins palliatifs dans le cas particulier du diagnostic prénatal à la place de l'association classique « feeticide-expulsion provoquée ». Ce type de prise en charge commence à se développer en France. Son analyse porte sur les obstacles à une diffusion plus large de ce type de soins par les équipes françaises.

Ce dossier aborde donc plusieurs sujets d'actualité qui prêtent à discussion. C'est pourquoi nous proposons à nos lecteurs de s'exprimer sur ces questions s'ils le souhaitent dans les prochains numéros dans une nouvelle rubrique intitulée « tribune libre ». Nous espérons que vous serez nombreux à réagir et à alimenter la discussion sur ce thème puis sur d'autres. 